\title{
Does Laparoscopic Adhesiolysis Reduce the Risk of Small Bowel Obstruction Related Readmissions and Reoperations Compared to Open Adhesiolysis?
}

\author{
Jin Hyung Park, M.D., Dong Jin Kim, M.D., Ph.D., Jung Hyun Park, M.D., Ph.D. \\ Department of Surgery, College of Medicine, The Catholic University of Korea, Seoul, Korea
}

\begin{abstract}
Purpose: The present study aimed to assess the safety and efficacy of laparoscopic adhesiolysis in decreasing recurrent episodes of small bowel obstruction (SBO) compared to that of the conventional open procedure.

Methods: Among 373 patients who visited our emergency department from January 2000 to July 2018 due to small bowel obstruction, 67 patients who underwent adhesiolysis were included in this study. Eighteen and 49 patients comprised the open adhesiolysis (OA) and laparoscopic adhesiolysis (LA) groups, respectively. Clinical demographics, computed tomography (CT) findings, laboratory results, and perioperative outcomes were compared. Further, the long-term follow-ups of SBO related re-admissions and re-operations were also compared.
\end{abstract}

Results: Preoperative baseline data, pain characteristics, laboratory findings, and ileus-related CT findings showed no significant difference between the two groups. LA was related to less blood loss and complications, along with early bowel movement recovery. Similarly, fewer SBO-related re-admissions [OA vs. LA=8 (44.4\%) vs. $3(6.1 \%), p=0.001$ ] and re-operations [OA vs. LA=3 (16.7\%) vs. $1(2.0 \%), p=0.025]$ were observed in LA compared to OA.

Conclusion: LA is a safer and more feasible procedure for SBO treatment compared to OA. This procedure showed a reduction in SBO-related re-admission and re-operation rates.

Keywords: Adhesion, Ileus, Laparoscopy, Adhesiolysis, Complication

\author{
Received October 30, 2019 \\ Revised 1st January 4, 2020 \\ 2nd February 25, 2020 \\ 3rd March 21, 2020 \\ Accepted March 26, 2020 \\ Corresponding author \\ Jung Hyun Park \\ Division of Gastrointestinal Surgery, \\ Department of Surgery, Eunpyeong \\ St. Mary's Hospital, College of \\ Medicine, The Catholic University of \\ Korea, 1021 Tongil-ro, Eunpyeong- \\ gu, Seoul 03312, Korea \\ Tel: +82-2-2030-4646 \\ Fax: +82-2-2030-4647 \\ E-mail: angle4901@gmail.com \\ ORCID:
}

https://orcid.org/0000-0003-2693-0655

\section{INTRODUCTION}

Acute small bowel obstruction ( $\mathrm{SBO}$ ) is one of the most common causes requiring emergency admission and surgery. ${ }^{1}$ Initial assessment and planning of when and how to perform surgical intervention are the most important aspects in managing SBO. Moreover, the chosen surgical approach — such as laparoscopy or open adhesiolysis - is another matter entirely. Laparoscopic procedures have become common throughout the intra-abdominal surgical field during the past two decades.
Thus, laparoscopic adhesiolysis has also become popular and widely studied. ${ }^{2-5}$

In general, the laparoscopic procedure has many benefits compared to open surgery including less postoperative pain, early recovery, and fewer complications in many fields of intra-abdominal surgery. ${ }^{4,67}$ However, questions remain regarding whether these advantages could be adopted to laparoscopic adhesiolysis for SBO. The risk of unintended bowel injury or time delay due to a narrow procedural space are important considerations. ${ }^{8,9}$ 
While strangulated or nearly strangulated SBO needs urgent surgical intervention, surgeons tend to delay surgical treatment for SBO in case of an equivocal situation. This is done to avoid additional adhesion formation after adhesiolysis which can be the cause of recurrent SBO. If laparoscopic adhesiolysis can be adopted safely, additional adhesions may be minimized and surgical intervention can be actively performed for these patients. From this point of view, proving the safety and feasibility of laparoscopic adhesiolysis compared to open adhesiolysis could deliver an important clinical value.

In this study, we aimed to compare not only the postoperative outcomes, but also the prevalence of $\mathrm{SBO}$-related readmission and re-operation rates between laparoscopic and open adhesiolysis.

\section{MATERIALS AND METHODS}

Our study involved patients diagnosed with SBO who underwent operation at Yeouido St. Mary's Hospital and St. Paul Hospital between January 2000 and July 2018. Among the 373 patients who visited the emergency department due to small bowel obstruction, 77 patients underwent adhesiolysis and were included in our study.

In our medical center, SBO was diagnosed based on the presence of small bowel dilatation and the characteristic "stepladder sign" on radiograph. In addition, SBO was diagnosed when one or more of the following three symptoms were found: abdominal pain, abdominal distension, and gas passage disturbance. We categorized the patients by dividing them into 2 groups, whether they underwent laparoscopic adhesiolysis (LA) or open adhesiolysis (OA) as an initial surgical approach. The patient selection flow chart is shown in Fig. 1. The diagnosis of SBO was based on the patient's history,

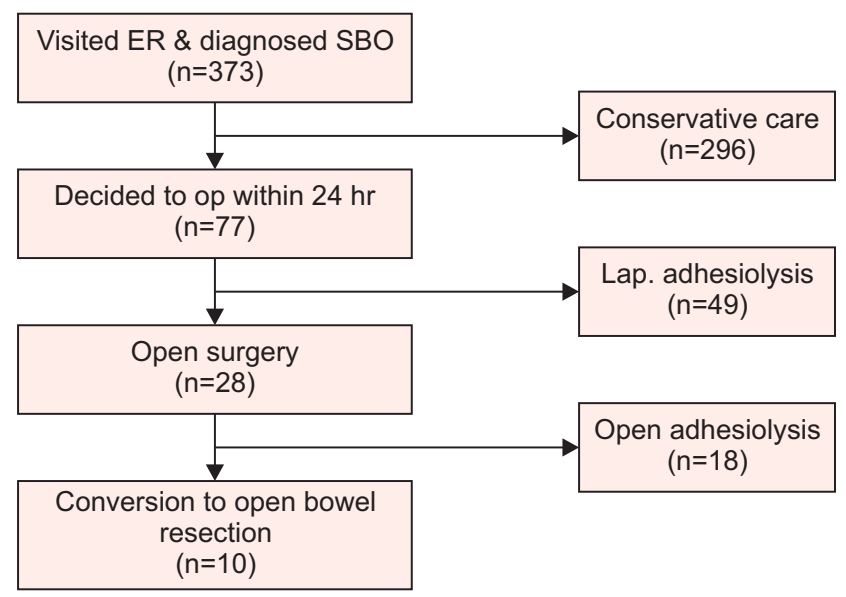

Fig. 1. Flowchart for patient selection $I S B O=$ small bowel obstruction; $\mathrm{ER}=$ emergency room). physical examination, laboratory findings, and imaging studies. We excluded 10 patients who underwent bowel resection. Initial preoperative data collection of patient's information included age, sex, body mass index (BMI), and operation history. The time from symptom onset was estimated. This was defined as the time from the beginning of abdominal pain to the emergency room visit. Initial laboratory examinations included blood cell count, neutrophil differential proportion, and serum $\mathrm{C}$-reactive protein (CRP) concentration. Conclusive computed tomography (CT) findings were represented by the presence of air fluid level, feces sign, pneumatosis, mesenteric edema, bowel twisting, perforation, free peritoneal fluid, mesenteric vessel thrombosis, and decreased bowel enhancement. The longest small bowel diameter and the aorto-peritoneal length were also considered. Additionally, transition zone (TZ) locations were found through $\mathrm{CT}$ imaging which were classified according to 10 areas, namely: (1) right hypochondriac region, (2) epigastric region, (3) left hypochondriac region (4) right lumbar region, (5) umbilical lesion, (6) left lumbar region, (7) right iliac lesion, (8) hypogastric lesion, (9) left iliac lesion, and (10) the previous wound site. The latter was identified when the $\mathrm{TZ}$ was attached to the peritoneum below the previous operation wound. Confirmation of each CT finding was made through formal readings by a radiologist from each hospital.

Perioperative data included operation time, surgical approach (LA versus OA), estimated blood loss, bowel resection performance, and adhesion type. The latter was classified by wound adhesion, band type, and dense adhesion.

Postoperative outcomes included length of hospital stay (LOS), first day of gas out, nasogastric (NG) tube removal day, liquid diet starting time, and presence of complications.

We also checked the incidence of SBO recurrence and reoperation through long-term follow-up. Recurrence was defined as the patient's hospital re-admission or admission to another hospital for the same cause. This was verified through outpatient follow-up or phone call. This study was approved by the institutional review board (IRB number: XC17REDI0060).

\section{Statistical analysis}

All statistical analyses were performed using PASW statistics, version 18.0 (IBM). Continuous variables were described as mean \pm standard deviation and compared using Student's ttest. Nominal variables were compared using chi-square and Fisher's exact tests. SBO-related recurrence was compared with the Kaplan-Meier model and the log-rank test. All statistical analyses were considered significant when the $p$ value was less than 0.05 . 


\section{RESULTS}

A total of 373 patients were diagnosed with SBO between January 2000 and July 2018. Among them, 296 received conservative care without surgery, while 77 patients underwent surgery. The decision was made within 24 hours. Twentyeight of them had open surgery, while 49 had laparoscopic surgery. Ten out of 28 patients underwent bowel resection and were excluded. Our analysis was based on the findings of the remaining 18 patients.

\section{Preoperative characteristics}

The patient demographics are summarized in Table 1. The
OA group had more female patients than the LA group. Other than this, there were no significant differences between the two groups with regards to age, BMI, pain characteristics, admission history, and type of previous operations.

The preoperative laboratory and computed tomography findings are summarized in Table 2.

Laboratory findings showed no differences between the two groups in terms of white blood cell count, segmented neutrophil count, lymphocyte count, albumin, creatine, CRP, and LDH levels.

On CT findings, the presence of air fluid, feces sign, pneumatosis, mesenteric edema, bowel twisting, perforation, free peritoneal fluid, mesenteric vessel thrombosis, decreased bowel enhancement, longest small bowel diameter, and the

Table 1. Patient demographics

\begin{tabular}{|c|c|c|c|c|}
\hline Variable & & $O A(n=18)$ & $L A(n=49)$ & $p$ value \\
\hline \multirow[t]{2}{*}{ Sex } & Male & $3(16.7 \%)$ & $25(50.1 \%)$ & 0.011 \\
\hline & Female & $15(83.3 \%)$ & $24(49 \%)$ & \\
\hline Age (year) & & $59.9 \pm 14.4$ & $55.7 \pm 19.3$ & 0.202 \\
\hline BMI $\left(\mathrm{kg} / \mathrm{m}^{2}\right)$ & & $22.2 \pm 4.7$ & $21.7 \pm 2.6$ & 0.421 \\
\hline \multirow[t]{3}{*}{ Pain characteristic } & Intermittent & $11(61.1 \%)$ & $25(51.0 \%)$ & 0.421 \\
\hline & Continuous & $7(38.9 \%)$ & $20(40.8 \%)$ & \\
\hline & Un known & 0 & $4(8.2 \%)$ & \\
\hline Time after symptom onset (hour) & & $22.3 \pm 25.2$ & $29.7 \pm 33.6$ & 0.205 \\
\hline \multirow[t]{5}{*}{ Ileus-admission history (count) } & 0 & 13 & 31 & 0.956 \\
\hline & 1 & 2 & 4 & \\
\hline & 2 & 2 & 4 & \\
\hline & 3 & 1 & 2 & \\
\hline & $\geq 4$ & 0 & 2 & \\
\hline \multirow[t]{7}{*}{ Type of previous operation_I } & None & $1(5.6 \%)$ & $9(16.9 \%)$ & 0.470 \\
\hline & UGI & $4(21.1 \%)$ & $8(15.1 \%)$ & \\
\hline & Small bowel & $2(10.5 \%)$ & $1(1.9 \%)$ & \\
\hline & LGI & $2(11.2 \%)$ & $4(7.5 \%)$ & \\
\hline & Appendix & $3(15.8 \%)$ & $12(22.6 \%)$ & \\
\hline & $\mathrm{OBGY}$ & $6(33.3 \%)$ & $16(31.2 \%)$ & \\
\hline & Hepatobiliary & $1(5.6 \%)$ & $3(5.6 \%)$ & \\
\hline \multirow[t]{2}{*}{ Type of previous operation_II } & Cancer & $4(23.5 \%)$ & $10(25.0 \%)$ & 0.906 \\
\hline & Benign & $13(76.5 \%)$ & $30(75.0 \%)$ & \\
\hline \multirow[t]{2}{*}{ Type of previous operation_III } & Laparoscopy & $1(5.9 \%)$ & $8(20.0 \%)$ & 0.235 \\
\hline & Open & $16(94.3 \%)$ & $30(75 \%)$ & \\
\hline
\end{tabular}

Continuous variables are expressed as mean \pm standard deviation; nominal variables are expressed as numbers $(\%) . O A=0$ open adhesiolysis; $L A=$ laparoscopic adhesiolysis; $\mathrm{BMI}=$ body mass index; $\mathrm{UGI}=$ upper gastrointestinal; $\mathrm{LGI}=$ lower gastrointestinal; $\mathrm{OBGYN}=$ obstetric gynecology . 
Table 2. Patient demographics, preoperative laboratory and computed tomography findings

\begin{tabular}{|c|c|c|c|}
\hline Variable & $\mathrm{OA}(\mathrm{n} 18)$ & LA (n 49) & $p$ value \\
\hline White Blood Cell (109/L) & $11.82 \pm 5.30$ & $11.48 \pm 4.20$ & 0.486 \\
\hline Segment neutrophils $(\%)$ & $79.22 \pm 9.60$ & $79.79 \pm 9.29$ & 0.752 \\
\hline Lymphocyte $(\%)$ & $14.74 \pm 5.47$ & $13.68 \pm 7.71$ & 0.485 \\
\hline Albumin (g/dL) & $4.41 \pm 0.44$ & $4.48 \pm 0.45$ & 0.798 \\
\hline Creatine (mg/dL) & $0.91 \pm 0.30$ & $1.03 \pm 0.58$ & 0.130 \\
\hline CRP (mg/dL) & $3.72 \pm 7.56$ & $10.14 \pm 17.53$ & 0.022 \\
\hline LDH (mg/dL) & $417.3 \pm 109.3$ & $440.3 \pm 111.6$ & 0.878 \\
\hline \multicolumn{4}{|l|}{ Computed tomography findings } \\
\hline Air-Fluid level & $18(100 \%)$ & $49(100 \%)$ & 67 \\
\hline Number of TZ point (0/1/2) & 0/15 (83.3\%)/3 (16.7\%) & $1(2.0 \%) / 44(89.7 \%) / 4(8.1 \%)$ & 0.511 \\
\hline Location of TZ point* & & & 0.154 \\
\hline 1/2/3 & $0 / 0 / 1(5.6 \%)$ & $0 / 0 / 3(6.1 \%)$ & \\
\hline $4 / 5 / 6$ & $1(5.6 \%) / 7(38.9 \%) / 2(11.1 \%)$ & $4(8.2 \%) / 8(16.3 \%) / 1(2.0 \%)$ & \\
\hline 7/8/9/10 & $4(22.2 \%) / 2(11.1 \%) / 1(5.6 \%)$ & $7(14.3 \%) / 7(14.3 \%) / 6(12.2 \%) / 12(24.5 \%)$ & \\
\hline Feces sign & $11(61.1 \%)$ & $32(65.3 \%)$ & 0.751 \\
\hline Pneumatosis & 0 & 0 & 67 \\
\hline Mesenteric edema & $6(33.3 \%)$ & $14(28.6 \%)$ & 0.706 \\
\hline Mesenteric twisting & $8(44.4 \%)$ & $14(28.6 \%)$ & 0.220 \\
\hline Free peritoneal Fluid & $9(50.0 \%)$ & $16(32.7 \%)$ & 0.193 \\
\hline Mesenteric vessel thrombosis & $1(5.6 \%)$ & $2(4.1 \%)$ & 0.796 \\
\hline Bowel enhance decline & $1(5.6 \%)$ & $6(12.2 \%)$ & 0.665 \\
\hline Bowel wall perforation & 0 & 0 & 67 \\
\hline Small bowel diameter $(\mathrm{cm})^{* *}$ & $37.1 \pm 7.6$ & $36.1 \pm 7.9$ & 0.202 \\
\hline AP length $(\mathrm{cm})^{* * *}$ & $58.9 \pm 16.6$ & $63.1 \pm 20.8$ & 0.613 \\
\hline
\end{tabular}

Continuous variables are expressed as mean \pm standard deviation; nominal variables are expressed as numbers $(\%) .0 A=$ open adhesiolysis; $L A=$ laparoscopic adhesiolysis; $\mathrm{TZ}=$ transition zone; $\mathrm{CRP}=\mathrm{C}$-reactive protein; $\mathrm{LDH}=$ lactate dehydrogenase. ${ }^{*}$ Location of TZ point: (1) Right hypochondriac region, (2) Epigastric region, (3) Left hypochondriac region, (4) Right lumbar region, (5) Umbilical lesion, (6) Left lumbar region, (7) Right iliac lesion, (8) Hypogastric lesion, (9) Left iliac lesion, and (10) Previous wound. ${ }^{*}$ Small bowel diameter: The longest mall bowel diameter in computed tomography. ${ }^{* * *} \mathrm{AP}$ length: The longest aorto-peritoneal diameter in computed tomography.

aorto-peritoneal length showed no significant differences between the two groups.

Most patients have at least one TZ in both groups. The most frequent locations were the periumbilical area and the previous wound site in the OA (38.9\%) and LA (24.5\%) groups, respectively. There was no significant difference in the $\mathrm{TZ}$ distribution between the two groups.

\section{Surgical outcomes}

In the LA group, estimated blood loss (EBL) was lower than the OA group (OA vs. $\mathrm{LA}=127.9 \pm 179.9 \mathrm{ml}$ vs. $36.7 \pm 62.6$ $\mathrm{ml}, p=0.001$ ). There was no significant difference in operation time between the two groups. (OA vs. LA=112.5 \pm 28.1 minutes vs. $71.8 \pm 37.8$ minutes, $p=0.121$ ). There were no intraoperative complications in both groups. Laparoscopic surgery was related to early recovery of bowel movement. The band type of adhesion was the most common in both groups $(72.2 \%$ in OA vs. $61.2 \%$ in LA). NG tube removal day was approximately one-third shorter in the LA group (1.1 \pm 1.0 vs. $3.56 \pm 3.36$, $p=0.001$. Similarly, time to gas passage was also shorter in the LA group (1.7 \pm 1.3 and $3.8 \pm 2.8, p=0.001)$ (Table 3). There were 
Table 3. Perioperative outcomes

\begin{tabular}{|c|c|c|c|c|}
\hline Variable & & $O A(n=18)$ & $\mathrm{LA}(\mathrm{n}=49)$ & $p$ value \\
\hline Operation time (minutes) & & $112.5 \pm 28.1$ & $71.8 \pm 37.8$ & 0.121 \\
\hline Estimated blood loss (ml) & & $127.9 \pm 179.9$ & $36.7 \pm 62.6$ & 0.001 \\
\hline \multirow[t]{2}{*}{ Initial Treatment } & Observation & $10(55.6 \%)$ & $9(18.4 \%)$ & \multirow[t]{2}{*}{0.003} \\
\hline & Surgery & $8(44.4 \%)$ & $40(80.6 \%)$ & \\
\hline \multirow[t]{3}{*}{ Adhesion type } & Band type & $13(72.2 \%)$ & $30(61.2 \%)$ & \multirow[t]{3}{*}{0.174} \\
\hline & Wound adhesion & $1(5.6 \%)$ & $12(24.5 \%)$ & \\
\hline & Dense adhesion & $4(22.2 \%)$ & $7(14.3 \%)$ & \\
\hline Time to liquid diet intake (days) & & $6.8 \pm 3.4$ & $3.1 \pm 2.3$ & 0.301 \\
\hline Length of hospital stay (days) & & $11.4 \pm 5.5$ & $7.2 \pm 4.7$ & 0.257 \\
\hline Time to NG tube removal (days) & & $3.56 \pm 3.36$ & $1.1 \pm 1.7$ & 0.001 \\
\hline Time to gas passage (days) & & $3.8 \pm 2.8$ & $1.7 \pm 1.3$ & 0.001 \\
\hline Complications & & $3(16.7 \%)$ & $2(4.7 \%)$ & 0.082 \\
\hline Re-admission & & $8(44.4 \%)$ & $3(6.1 \%)$ & 0.001 \\
\hline Re-operation & & $3(16.7 \%)$ & $1(2.0 \%)$ & 0.025 \\
\hline
\end{tabular}

Continuous variables are expressed as mean \pm standard deviation; nominal variables are expressed as numbers $(\%) . \mathrm{OA}=$ open adhesiolysis; $\mathrm{LA}=$ laparoscopic adhesiolysis; $N G$ = nasogastric.

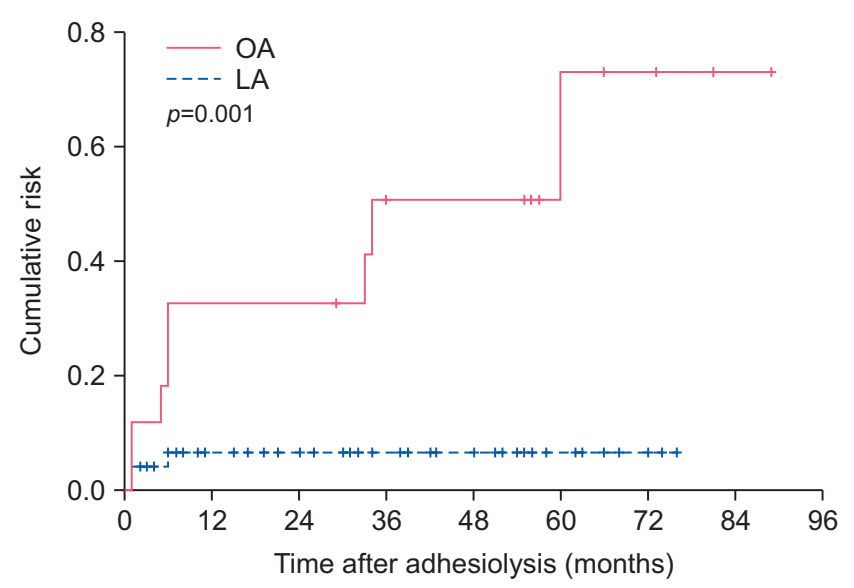

Fig. 2. Cumulative risk for small bowel obstruction related re-admission IOA = open adhesiolysis; $\mathrm{LA}=$ laparoscopic adhesiolysis).

no significant differences in liquid diet intake day and length of hospital stay between the two groups $(p=0.301,0.257)$. Postoperative complications occurred in 3 (ileus, pleural effusion, and ventral hernia) out of 18 patients in the OA group. In the LA group, 2 out of 49 patients had postoperative complications such as wound infection and umbilical hernia. However, the rate of postoperative complications was not significantly different between the OA and LA groups ( $16.7 \%$ vs. $4.7 \%$, $p=0.082$ ).
Most noticeable were the recurrence and re-operation rates which were significantly lower in the LA group (re-admissions: $44.4 \%$ vs. $6.1 \%, p=0.001$; re-operations: $16.7 \%$ vs. $2.0 \%$, $p=0.025$ ) (Table 3). The Kaplan-Meier analysis showed the significant difference in re-admission between both groups (log-rank test, $p=0.001$, Fig. 2). The median follow-up period for recurrence and re-operation was 36 months.

\section{DISCUSSION}

Our study showed that LA was associated with various postoperative advantages compared to $\mathrm{OA}$ in $\mathrm{SBO}$ treatment. LA showed better short-term surgical outcomes, and long-term effects on SBO recurrence and SBO-related readmissions and re-operations. Many studies have reported the benefits of laparoscopic approach for SBO such as less surgical infections, fewer morbidities, and fewer mortalities, ${ }^{10-13}$ However, there is still concern about the risk of bowel injury; hence, strict case selection is mandatory. ${ }^{9,14}$ In the current study, LA showed definite advantages in terms of less blood loss, shorter hospital stay, faster oral diet return, and shorter NG tube placement days.

LA also showed less postoperative complications compared to OA despite the lack of significance due to the small sample size in OA sampled patients. Although there was no significant difference between the two groups in our study, several prior 
reports have shown that LA has significantly fewer complications, shorter lengths of hospital stay, as well as reduced incidence of wound infection, pneumonia, and postoperative pain compared to OA. ${ }^{4}$ Because the pain is decreased, it can affect the improvement of the diaphragmatic function. Therefore, the incidence of complications due to general anesthesia such as pneumonia may be lower. ${ }^{10}$ As a result, the overall incidence of complications is lowered. Since dietary initiation is accelerated, this can directly affect the shortening of the hospitalization period.

On the other hand, LA can have a risk of unintended bowel injury during the introduction of trocars into a distended abdomen or through the intricate laparoscopic handling of the distended small bowel in a narrow space. It is also unsuitable in cases of dense adhesions or in patients with multiple medical comorbidities who present a greater anesthetic risk. ${ }^{9}$ Some studies have shown that when bowel injury occurs during LA, clinical outcomes are worse than if the patient had an open surgery initially. The risk of bowel injury may be diminished by following good surgical practices including the use of electrocautery, minimization of grasping the dilated bowel, manipulation of the bowel using atraumatic graspers, and handling the mesentery whenever possible. ${ }^{15}$

Surgeons planning a laparoscopic approach in patients with an SBO should be mindful of these risks and take measures to mitigate them where possible. Such measures might include opting for laparotomy in high-risk patients or being prepared to convert to laparotomy early. ${ }^{9}$

In this study, one of the most noticeable aspects of treating SBO patients is the recurrence rate. There was concern regarding the possibility of recurrence due to surgical treatment. We believed that the surgical approach to be employed would be a burden in determining the treatment method in patients with ileus. Therefore, the risk of ileus recurrence with LA was the key to SBO treatment. In our study, fewer ileus related re-admissions $(p=0.001)$ and re-operations $(p=0.025)$ were observed in the LA group compared to the OA group. Limited data exists regarding the recurrence or re-operation rate following adhesiolysis. Yao et al. (2017) compared the SBOrelated re-admission and re-operation rates between laparoscopic and open matched patients. ${ }^{16}$ In this study, SBO-related re-operation was higher in LA than OA. The authors suggest that the missed band may be the cause of recurrent SBO requiring re-operation. However, in our procedure, we explored the whole abdominal cavity and found adhesions where the potential band might have been removed. Theoretically, the longer the peritoneum is incised, the more intra-abdominal adhesion develops. For this reason, we experienced more readmissions and re-operations in OA patients.

The safety and less recurrence parameters of LA might influence the strategy for SBO management. In most cases, surgical intervention might be the last resort because of the adhesiolysis risk and fear of recurrence. In our study, $40 \mathrm{pa}^{-}$ tients underwent early surgical approach in the LA group. In those subgroups, hospital stay, day of NG tube removal, flatus passage, operation time, and estimated blood loss were $6.7 \pm 4.2$ days, $1.1 \pm 1.7$ days, $1.6 \pm 1.3$ days, $71.8 \pm 37.0$ minutes, and 40.8 $\pm 68.0 \mathrm{ml}$, respectively. One SBO-related re-operation was needed for a single patient. From these results, we can suggest that the early approach with LA is safe and helpful in minimizing the total hospital stay and early recovery. One study also showed that early LA application was helpful in early recovery and shortening of the fasting period. Additionally, less radiologic exams and hospital stays were required. ${ }^{17}$

This study had several limitations. First, because our study cohorts were from two different hospitals throughout a 17-year-long period, the surgical strategy for all patients may have been heterogenic. Second, there was selection bias between the OA and LA groups. Logically speaking, the more severe cases were included in the OA group. To minimize the selection bias, we excluded ten patients who needed small bowel resection. In addition, we analyzed almost all the characteristics of the $\mathrm{CT}$ and laboratory findings which could reflect the SBO status. There were no significant differences between the two groups.

\section{CONCLUSION}

LA is a safer and more feasible procedure in SBO treatment compared to OA. Moreover, it can minimize further adhesion formation after adhesiolysis and decrease ileus-related readmission and re-operation rates.

\section{ORCID}

Jin Hyung Park, https://orcid.org/0000-0001-7846-1112

Dong Jin Kim, https://orcid.org/0000-0001-5103-5607

Jung Hyun Park, https://orcid.org/0000-0003-2693-0655

\section{AUTHORS' CONTRIBUTIONS}

Conceptualization: Jung Hyun Park. Formal analysis: Dong Jin Kim. Methodology: Jung Hyun Park, Dong Jin Kim. Writing-original draft: Jin Hyung Park, Dong Jin Kim. Writingreview and editing: Dong Jin Kim, Jung Hyun Park.

\section{CONFLICT OF INTEREST}

None. 


\section{FUNDING}

None.

\section{ACKNOWLEDGMENTS}

None.

\section{REFERENCES}

1) Coe TM, Chang DC, Sicklick JK. Small bowel volvulus in the adult populace of the United States: results from a populationbased study. Am J Surg 2015;210:201-210.e202.

2) Catena F, Di Saverio S, Kelly MD, et al. Bologna Guidelines for Diagnosis and Management of Adhesive Small Bowel Obstruction (ASBO): 2010 Evidence-Based Guidelines of the World Society of Emergency Surgery. World J Emerg Surg 2011;6:5.

3) Kelly KN, Iannuzzi JC, Rickles AS, Garimella V, Monson JR, Fleming FJ. Laparotomy for small-bowel obstruction: first choice or last resort for adhesiolysis? A laparoscopic approach for smallbowel obstruction reduces 30-day complications. Surg Endosc 2014;28:65-73.

4) Patel R, Borad NP, Merchant AM. Comparison of outcomes following laparoscopic and open treatment of emergent small bowel obstruction: an 11-year analysis of ACS NSQIP. Surg Endosc 2018;32:4900-4911.

5) Paajanen $P$, Fagerstrom A, Paajanen H. Laparoscopic Adhesiolysis in Chronic Abdominal Pain: 15-Year Follow-up Study. J Clin Gastroenterol 2018;52:e32-e36.

6) Kim W, Kim HH, Han SU, et al. Decreased Morbidity of Laparoscopic Distal Gastrectomy Compared With Open Distal Gastrectomy for Stage I Gastric Cancer: Short-term Outcomes From a Multicenter Randomized Controlled Trial (KLASS-01). Ann Surg 2016;263:28-35.

7) Turcu F, Arnautu O, Copaescu C. Adhesiolysis-Related Challenges for Laparoscopic Procedures after Ventral Hernia Repair with Intraperitoneal Mesh. Chirurgia (Bucur) 2019;114:39-47.
8) Szeliga J, Jackowski M. Laparoscopy in small bowel obstruction current status - review. Wideochir Inne Tech Maloinwazyjne 2017;12:455-460.

9) Behman R, Nathens AB, Byrne JP, Mason S, Look Hong N, Karanicolas PJ. Laparoscopic Surgery for Adhesive Small Bowel Obstruction Is Associated With a Higher Risk of Bowel Injury: A Population-based Analysis of 8584 Patients. Ann Surg 2017;266:489-498.

10) Schwenk W, Bohm B, Witt C, Junghans T, Grundel K, Muller JM. Pulmonary function following laparoscopic or conventional colorectal resection: a randomized controlled evaluation. Arch Surg 1999;134:6-12; discussion 13.

11) Lin H, Li J, Xie Z, Zhang W, Lv X. Laparoscopic Versus Open Adhesiolysis for Small Bowel Obstruction: A Single-Center Retrospective Case-Control Study. Surg Laparosc Endosc Percutan Tech 2016;26:244-247.

12) Mancini GJ, Petroski GF, Lin WC, Sporn E, Miedema BW, Thaler K. Nationwide impact of laparoscopic lysis of adhesions in the management of intestinal obstruction in the US. J Am Coll Surg 2008;207:520-526.

13) Ghosheh B, Salameh JR. Laparoscopic approach to acute small bowel obstruction: review of 1061 cases. Surg Endosc 2007;21:1945-1949.

14) Di Saverio S, Birindelli A, Broek RT, Davies JR, Mandrioli M, Sallinen V. Laparoscopic adhesiolysis: not for all patients, not for all surgeons, not in all centres. Updates Surg 2018;70:557-561.

15) Levard H, Boudet MJ, Msika S, et al. Laparoscopic treatment of acute small bowel obstruction: a multicentre retrospective study. ANZ J Surg 2001;71:641-646.

16) Yao S, Tanaka E, Matsui $Y$, et al. Does laparoscopic adhesiolysis decrease the risk of recurrent symptoms in small bowel obstruction? A propensity score-matched analysis. Surg Endosc 2017;31:5348-5355.

17) Mazzetti CH, Serinaldi F, Lebrun E, Lemaitre J. Early laparoscopic adhesiolysis for small bowel obstruction: retrospective study of main advantages. Surg Endosc 2018;32:2781-2792. 